\title{
Characterizations of Certain Recently Introduced Distributions
}

\author{
G.G. Hamedani \\ Department of Mathematics, Statistics and Computer Science \\ Marquette University, Milwaukee, WI 53201-1881, USA \\ g.hamedani@mu.edu \\ Received 5 January 2012 \\ Accepted 6 June 2012
}

\begin{abstract}
Various characterizations of the Beta Weibull Geometric distribution of Bidram et al. (2011), the New Generalized Exponential distribution of Bidram et al. (2012), and the Gamma Exponentiated Weibull distribution of Pinho et al. (2012) are presented. These characterizations are based on: (i) a simple relationship between two truncated moments (ii) certain functions of the $n^{\text {th }}$ order statistic, and (iii) certain functions of the $1^{\text {st }}$ order statistics.
\end{abstract}

Keywords: Beta exponential distribution; Beta Weibull geometric distribution; Gamma exponentiated distribution; Gamma geometric distribution; Generalized exponential distribution; Weibull geometric distribution.

\section{Introduction}

It is widely known that the problem of characterizing a distribution is an important problem which has recently attracted the attention of many researchers. Thus, various characterizations have been established in many different directions. The present work deals with the characterizations of three continuous univariate distributions: Beta Weibull Geometric (BWG), New Generalized Exponential Geometric (NGEG) introduced by Bidram et al. [1,2] and Gamma Exponentiated Weibull (GEW) of Pinho et al. [16]. These characterizations are based on: (i) a simple relationship between two truncated moments, (ii) certain functions of the $n^{\text {th }}$ order statistic, and (iii) certain functions of the $1^{\text {st }}$ order statistic. The BWG distribution is a generalization of Weibull Geometric (WG) distribution proposed by Ortega et al. [15]. The BWG distribution is a five-parameter distribution which depends on scale, shape and, what we call, mixing parameters. This distribution is considered to be a suitable distribution for modeling monotone or unimodal failure rates. We refer the reader to Bidram et al. [1] for a detailed discussion as well as applications of BWG distribution. Bidram et al. [2] introduced the NGEG distribution based on the maximum of $N$ i.i.d. (independent and identically distributed) random variables $X_{j}, j=1,2, \ldots, N$ whose common distribution is a generalized exponential distribution, where $N$ has a geometric distribution and is independent of $X_{j}$ 's. This resulted in, as pointed out by Bidram et al. [2], a three-parameter distribution with applications to parallel systems with i.i.d. components as well as other biological organisms or industry units. Again, we 
refer the reader to Bidram et al. [2] for a detailed discussion as well as applications of NGEG distribution. The GEW distribution is a four-parameter distribution which includes several well-known distributions as special cases. It is shown in [16] that its density function may be expressed as an infinite linear combination of Weibull densities. For further details and the domain of applicability of GEW distribution, we refer the reader to Pinho et al. [16].

An investigator will be vitally interested to know if their model fits the requirements of the BWG or NGEG or GEW distribution. To this end, one will depend on the characterizations of these distributions which provide conditions under which the underlying distribution is indeed one of these distributions. Although in many applications an increase in the number of parameters provides a more suitable model, in characterization problems a lower number of parameters (without seriously affecting the suitability of the model) is mathematically more appealing (see Glänzel and Hamedani [9]). In view of this observation, we reduce the parameters of BWG distribution to four in our characterization of this distribution based on the $n^{\text {th }}$ order statistic.

The $p d f$ (probability density function) and $c d f$ (cumulative distribution function) corresponding to BWG distribution are given, respectively, by

$$
f(x)=f(x ; a, b, \alpha, \beta, p)=\frac{(1-p)^{b} \alpha \beta(\beta x)^{\alpha-1} e^{-b(\beta x)^{\alpha}}\left(1-e^{-(\beta x)^{\alpha}}\right)^{a-1}}{B(a, b)\left(1-p e^{-(\beta x)^{\alpha}}\right)^{(a+b)}}, x>0,
$$

and

$$
F(x)=F(x ; a, b, \alpha, \beta, p)=\frac{1}{B(a, b)} \int_{0}^{G(x)} t^{a-1}(1-t)^{b-1} d t, \quad x \geq 0,
$$

where $a>0, b>0, \alpha>0, \beta>0$ and $p \in(0,1)$ are parameters $B(a, b)=\int_{0}^{1} t^{a-1}(1-t)^{b-1} d t$ and $G(x)$ is a $c d f$ corresponding to WG distribution given by

$$
G(x)=\left(1-e^{-(\beta x)^{\alpha}}\right)\left(1-p e^{-(\beta x)^{\alpha}}\right)^{-1}, \quad x \geq 0 .
$$

The main parameters $\alpha$ and $\beta$ are shape and scale and $p$ is mixing parameters, respectively.

Note that for $a=b=1, F(x)=G(x)$ and hence WG distribution is a special case of BWG distribution.

The $p d f$ and $c d f$ of the generalized exponential distribution are

$$
\left.\begin{array}{ll}
f_{1}(x ; \alpha, \beta)=\alpha \beta e^{-\beta x}\left(1-e^{-\beta x}\right)^{\alpha-1}, & x>0, \\
F_{1}(x ; \alpha, \beta)=\left(1-e^{-\beta x}\right)^{\alpha}, & x \geq 0 .
\end{array}\right\} \quad \alpha>0, \beta>0
$$

Bidram et al. [2] assume that $X_{j}, j=1,2, \ldots, N$ are $N$ i.i.d. random variables with $c d f F_{1}$, where $N$ has a geometric distribution with parameter $p \in(0,1)$ and is independent of $X_{j}$ 's. Then, they define 
$X=\max \left\{X_{j}: j=1,2, \ldots, N\right\}$ which has $p d f$ and $c d f$ given, respectively, by

$$
f(x)=f(x ; \alpha, \beta, p)=\frac{(1-p) \alpha \beta e^{-\beta x}\left(1-e^{-\beta x}\right)^{a-1}}{\left\{1-p\left(1-e^{-\beta x}\right)^{\alpha}\right\}^{2}}, \quad x>0,
$$

and

$$
F(x)=F(x ; \alpha, \beta, p)=\frac{(1-p)\left(1-e^{-\beta x}\right)^{a}}{1-p\left(1-e^{-\beta x}\right)^{\alpha}}, \quad x \geq 0,
$$

where $\alpha>0, \beta>0$ and $p \in(0,1)$ are shape, scale and mixing parameters respectively. Bidram et al. [2] call this distribution NGEG.

The $p d f$ and $c d f$ of GEW distribution are given, respectively, by

$$
\begin{aligned}
f(x) & =f(x ; \alpha, \beta, k, \delta) \\
& =\frac{k \alpha^{\delta}}{\beta \Gamma(\delta)}\left(\frac{x}{\beta}\right)^{k-1} e^{-\left(\frac{x}{\beta}\right)^{k}}\left[1-e^{-\left(\frac{x}{\beta}\right)^{k}}\right]^{\alpha-1}\left\{-\ln \left[1-e^{-\left(\frac{x}{\beta}\right)^{k}}\right]\right\}^{\delta-1}, \quad x>0,
\end{aligned}
$$

and

$$
F(x)=F(x ; \alpha, \beta, k, \delta)=1-\frac{\gamma\left\{-\ln \left[1-e^{-\left(\frac{x}{\beta}\right)^{k}}\right]^{\alpha}, \delta\right\}}{\Gamma(\delta)}, \quad x \geq 0
$$

where the parameters $\alpha, \beta, k, \delta$ are all positive and $\gamma(x, \delta)=\int_{0}^{x} u^{\delta-1} e^{-u} d u$.

\section{Characterization Results}

As we mentioned in the Introduction, the BWG, NGEG and GEW distributions (and their special cases listed in [1,2] and [16] respectively) may have potential applications in many fields of studies. So, an investigator will be vitally interested to know if their model fits the requirements of the BWG or NGEG or GEW distribution. To this end, one will depend on the characterizations of these distributions which provide conditions under which the underlying distribution is indeed one of these distributions.

\subsection{Characterization based on two truncated moments}

In this subsection we present characterizations of the BWG, NGEG and GEW distributions in terms of a simple relationship between two truncated moments. We like to mention here the works of Galambos and Kotz [3], Kotz and Shanbahag [14], Glänzel [4-6], Glänzel et al. [7, 8], Glänzel and Hamedani [9] and Hamedani [10-12] in this direction. Our characterization results presented here will employ an interesting result due to Glänzel [5] (Theorem G below).

Theorem G. Let $(\Omega, \mathscr{F}, \mathbf{P})$ be a given probability space and let $H=[a, b]$ be an interval for some $a<b(a=-\infty, b=\infty$ might as well be allowed). Let $X: \Omega \rightarrow H$ be a continuous random variable 
with the distribution function $F$ and let $g$ and $h$ be two real functions defined on $H$ such that

$$
\mathbf{E}[g(x) \mid X \geq x]=\mathbf{E}[h(x) \mid X \geq x] \eta(x), \quad x \in H,
$$

is defined with some real function $\eta$. Assume that $g, h \in C^{1}(H), \eta \in C^{2}(H)$ and $F$ is twice continuously differentiable and strictly monotone function on the set $H$. Finally, assume that the equation $h \eta=g$ has no real solution in the interior of $H$. Then $F$ is uniquely determined by the functions $g$, $h$ and $\eta$, particularly

$$
F(x)=\int_{a}^{x} C\left|\frac{\eta^{\prime}(u)}{\eta(u) h(u)-g(u)}\right| \exp (-s(u)) d u
$$

where the function $s$ is a solution of the differential equation $s^{\prime}=\frac{\eta^{\prime} h}{\eta h-g}$ and $C$ is a constant, chosen to make $\int_{H} d F=1$.

Remarks 2.1.1. (a) In Theorem G, the interval $H$ need not be closed.

(b) The goal is to have the function $\eta$ as simple as possible. For a more detailed discussion on the choice of $\eta$, we refer the reader to Glänzel and Hamedani [9] and Hamedani [10-12].

Proposition 2.1.2. Let $X: \Omega \rightarrow(0, \infty)$ be a continuous random variable and let

$$
h(x)=e^{(b-a)(\beta x)^{\alpha}}\left(1-e^{-(\beta x)^{\alpha}}\right)^{1-a}\left(1-p e^{-(\beta x)^{\alpha}}\right)^{a+b}
$$

and

$$
g(x)=\left(1-e^{-(\beta x)^{\alpha}}\right)^{1-a}\left(1-p e^{-(\beta x)^{\alpha}}\right)^{a+b}
$$

for $x \in(0, \infty)$. The pdf of $X$, with $a \neq b$, is (1) if and only if the function $\eta$ defined in Theorem $G$ has the form

$$
\eta(x)=\frac{a}{b} e^{(a-b)(\beta x)^{\alpha}}, \quad x>0 .
$$

Proof. Let $X$ have $p d f(1)$, then

$$
(1-F(x)) \mathbf{E}[h(x) \mid X \geq x]=\frac{(1-p)^{b}}{a B(a, b)} e^{-a(\beta x)^{\alpha}}, \quad x>0,
$$

and

$$
(1-F(x)) \mathbf{E}[g(x) \mid X \geq x]=\frac{(1-p)^{b}}{a B(a, b)} e^{-b(\beta x)^{\alpha}}, \quad x>0,
$$

and finally

$$
\eta(x) h(x)-g(x)=\frac{a-b}{b}\left[\left(1-e^{-(\beta x)^{\alpha}}\right)^{1-a}\left(1-p e^{-(\beta x)^{\alpha}}\right)^{a+b}\right] \neq 0, \text { for } x>0 .
$$

Conversely, if $\eta$ is given as above, then

$$
s^{\prime}(x)=\frac{\eta^{\prime}(x) h(x)}{\eta(x) h(x)-g(x)}=a \alpha \beta(\beta x)^{\alpha-1}, \quad x>0,
$$


and hence

$$
s(x)=a(\beta x)^{\alpha}, \quad x>0 .
$$

Now, in view of Theorem G, $X$ has $c d f(2)$ and $p d f(1)$.

Corollary 2.1.3. Let $X: \Omega \rightarrow(0, \infty)$ be a continuous random variable and let $h(x)$ be as in Proposition 2.1.2. The pdf of $X$ with $a \neq b$, is (1) if and only if there exist functions $g$ and $\eta$ defined in Theorem $G$ satisfying the differential equation

$$
\frac{\eta^{\prime}(x) h(x)}{\eta(x) h(x)-g(x)}=a \alpha \beta(\beta x)^{\alpha-1}, \quad x>0 .
$$

Remarks 2.1.4. (i) The general solution of the differential equation in Corollary 2.1 .3 is

$$
\eta(x)=e^{a(\beta x)^{\alpha}}\left[-\int g(x) a \alpha \beta(\beta x)^{\alpha-1} e^{-b(\beta x)^{\alpha}}\left(1-e^{-(\beta x)^{\alpha}}\right)^{a-1}\left(1-p e^{-(\beta x)^{\alpha}}\right)^{-(a+b)} d x+D\right],
$$

for $x>0$, where $D$ is a constant. One set of appropriate functions is given in Proposition 2.1.2 with $D=0$.

(ii) In Proposition 2.1.2 we assume $a \neq b$. This condition can be removed with a minor change in the expressions for functions $h$ and $g$ as given in the following Corollary.

Corollary 2.1.5. Let $X: \Omega \rightarrow(0, \infty)$ be a continuous random variable and let

$$
h(x)=e^{(b-1)(\beta x)^{\alpha}}\left(1-e^{-(\beta x)^{\alpha}}\right)^{1-a}\left(1-p e^{-(\beta x)^{\alpha}}\right)^{a+b-2}
$$

and

$$
g(x)=e^{(b-1)(\beta x)^{\alpha}}\left(1-e^{-(\beta x)^{\alpha}}\right)^{1-a}\left(1-p e^{-(\beta x)^{\alpha}}\right)^{a+b}
$$

for $x \in(0, \infty)$. The pdf of $X$ is (1) if and only if the function $\eta$ defined in Theorem $G$ is of the form

$$
\eta(x)=\left(1-p e^{-(\beta x)^{\alpha}}\right), \quad x>0 .
$$

Proof. Is similar to that of Proposition 2.1.2 with a little more work to recover $s(x)$ properly from $s^{\prime}(x)$.

Remarks 2.1.6. (i) Clearly there are other triplets of functions $(h, g, \eta)$ satisfying the conditions of Theorem G. We presented two such triplets in Proposition 2.1.2 and Corollary 2.1.5. (ii) As we mentioned earlier, for $a=b=1 \mathrm{BWG}$ distribution reduces to WG distribution. In this case, Corollary 2.1.5 will reduce to Proposition 2.1.2 of Hamedani and Ahsanullah [14].

Proposition 2.1.7. Let $X: \Omega \rightarrow(0, \infty)$ be a continuous random variable and let $h(x)=1$ and $g(x)=\left\{1-p\left(1-e^{-\beta x}\right)^{\alpha}\right\}^{2}$ for $x \in(0, \infty)$. The pdf of $X$ is (3) if and only if the function $\eta$ defined in Theorem $G$ has the form

$$
\eta(x)=(1-p)\left\{1-p\left(1-e^{-\beta x}\right)^{\alpha}\right\}, \quad x>0 .
$$


Proof. Is similar to that of Proposition 2.1.2 with an observation that for the converse, in recovering $s(x)$ from $s^{\prime}(x)$, one may use $1-p=\left\{1-p\left(1-e^{-\beta x}\right)^{\alpha}\right\}-p\left\{1-\left(1-e^{-\beta x}\right)^{\alpha}\right\}$.

Proposition 2.1.8. Let $X: \Omega \rightarrow(0, \infty)$ be a continuous random variable and let

$$
h(x)=\left[1-e^{-\left(\frac{x}{\beta}\right)^{k}}\right]^{2-\alpha}\left\{-\ln \left[1-e^{-\left(\frac{x}{\beta}\right)^{k}}\right]\right\}^{1-\delta}
$$

and

$$
g(x)=\left[1-e^{-\left(\frac{x}{\beta}\right)^{k}}\right]^{4-\alpha}\left\{-\ln \left[1-e^{-\left(\frac{x}{\beta}\right)^{k}}\right]\right\}^{1-\delta}
$$

for $x \in(0, \infty)$. The pdf of $X$ is (5) if and only if the function $\eta$ defined in Theorem $G$ has the form

$$
\eta(x)=2^{-1}\left\{1+\left[1-e^{-\left(\frac{x}{\beta}\right)^{k}}\right]^{2}\right\}, \quad x>0 .
$$

Remark 2.1.9. A corollary and a remark similar to Corollary 2.1 .3 and Remark 2.1.4 (part (i)) can be stated for NGEG and GEW as well.

\subsection{Characterization of BWG and NGEG based on truncated moment of certain functions of the $n^{\text {th }}$ order statistic}

Let $X_{1: n} \leq X_{2: n} \leq \cdots \leq X_{n: n}$ be $n$ order statistics from a continuous $c d f F$. We present here two characterizations base on certain functions of the $n^{\text {th }}$ order statistic. We take $b=1$ in (1) and hence $F(x)=[G(x)]^{a}$. So, $F(x)$ will be an exponentiated distribution with base $c d f G$ and the exponent parameter $a>0$. Our characterizations of BWG (for $a \neq b$ ) and NGEG distributions here will be a consequence of the following proposition, which is similar to the one appeared in our previous work. We give a brief proof of it here for the sake of completeness.

Proposition 2.2.1. Let $X: \Omega \rightarrow(0, \infty)$ be a continuous random variable with cdf $F$. Let $\psi(x)$ and $q(x)$ be two differentiable functions on $(0, \infty)$ such that $\lim _{x \rightarrow 0} \psi(x)[F(x)]^{n}=0$ and $\int_{0}^{\infty} \frac{q^{\prime}(t)}{[\psi(t)-q(t)]} d t=\infty$. Then

$$
E\left[\psi\left(X_{n: n}\right) \mid X_{n: n}<t\right]=q(t), \quad t>0,
$$

implies

$$
F(x)=\exp \left\{-\int_{x}^{\infty} \frac{q^{\prime}(t)}{n[\psi(t)-q(t)]} d t\right\}, \quad x \geq 0
$$


Proof. If (7) holds, then using integration by parts on the left hand side of (7) and the assumption $\lim _{x \rightarrow 0} \psi(x)[F(x)]^{n}=0$, we have

$$
\int_{0}^{t} \psi^{\prime}(x)(F(x))^{n} d x=[\psi(t)-q(t)](F(t))^{n} .
$$

Differentiating both sides of the above equation with respect to $t$, we arrive at

$$
\frac{f(t)}{F(t)}=\frac{q^{\prime}(t)}{n[\psi(t)-q(t)]}, \quad t>0
$$

Now, integrating (9) from $x$ to $\infty$, we have, in view of $\int_{0}^{\infty} \frac{q^{\prime}(t)}{[\psi(t)-q(t)]} d t=\infty$, a $c d f F$ given by (8).

Remarks 2.2.2. (a) Taking, e.g., $\psi(x)=\left(1-p e^{-(\beta x)^{\alpha}}\right)^{n a+1}$ and $q(x)=(1-p)\left(1-p e^{-(\beta x)^{\alpha}}\right)^{n a}$ in Proposition 2.2.1, equation (9) will be

$$
\frac{f(x)}{F(x)}=\frac{a(1-p) \alpha \beta(\beta x)^{\alpha-1} e^{-b(\beta x)^{\alpha}}}{\left(1-p e^{-(\beta x)^{\alpha}}\right)\left(1-e^{-(\beta x)^{\alpha}}\right)} .
$$

Now, replacing $(1-p)$ with $\left[\left(1-p e^{-(\beta x)^{\alpha}}\right)-p\left(1-e^{-(\beta x)^{\alpha}}\right)\right]$ in (10) we have

$$
\frac{f(x)}{F(x)}=a\left[\frac{\alpha \beta(\beta x)^{\alpha-1} e^{-b(\beta x)^{\alpha}}}{1-e^{-(\beta x)^{\alpha}}}-\frac{p \alpha \beta(\beta x)^{\alpha-1} e^{-b(\beta x)^{\alpha}}}{1-p e^{-(\beta x)^{\alpha}}}\right],
$$

from which, in view of (8), we have $F(x)=[G(x)]^{a}$, where $G(x)$ is $c d f$ corresponding to WG distribution.

(b) Taking, e.g., $\psi(x)=\left\{1-p\left(1-e^{-\beta x}\right)^{\alpha}\right\}^{n+1}$ and $q(x)=\left\{1-p\left(1-e^{-\beta x}\right)^{\alpha}\right\}^{n}$ in Proposition 2.2.1, equation (9) will be

$$
\frac{f(x)}{F(x)}=\frac{\alpha \beta e^{-\beta x}}{\left\{1-p\left(1-e^{-\beta x}\right)^{\alpha}\right\}\left(1-e^{-\beta x}\right)}
$$

Rewriting (11) we have

$$
\frac{f(x)}{F(x)}=\left[\frac{p \alpha \beta e^{-\beta x}\left(1-e^{-\beta x}\right)^{\alpha-1}}{1-p\left(1-e^{-\beta x}\right)^{\alpha}}+\frac{\alpha \beta e^{-\beta x}\left(1-e^{-\beta x}\right)^{\alpha-1}}{\left(1-e^{-\beta x}\right)^{\alpha}}\right],
$$

from which, in view of (8), we have $F(x)=\frac{(1-p)\left(1-e^{-\beta x}\right)^{\alpha}}{1-p\left(1-e^{-\beta x}\right)^{\alpha}}$.

\subsection{Characterization of NGEG and GEW based on truncated moment of certain functions of the $1^{\text {st }}$ order statistic}

Our characterizations of NGEG and GEW distributions here will be a consequence of the following proposition, which again is similar to another one appeared in our previous work. We give a brief proof of it here for the sake of completeness. 
Proposition 2.3.1. Let $X: \Omega \rightarrow(0, \infty)$ be a continuous random variable with cdf $F$. Let $\psi_{1}(x)$ and $q_{1}(x)$ be two differentiable functions on $(0, \infty)$ such that $\lim _{x \rightarrow \infty} \psi_{1}(x)[1-F(x)]^{n}=0$ and $\int_{0}^{\infty} \frac{-q_{1}^{\prime}(t)}{\left[\psi_{1}(t)-q_{1}(t)\right]} d t=\infty$. Then

$$
E\left[\psi_{1}\left(X_{1: n}\right) \mid X_{1: n}>t\right]=q_{1}(t), \quad t>0
$$

implies

$$
F(x)=1-\exp \left\{\int_{0}^{x} \frac{q_{1}^{\prime}(t)}{n\left[\psi_{1}(t)-q_{1}(t)\right]} d t\right\}, \quad x \geq 0 .
$$

Proof. If (12) holds, then using integration by parts on the left hand side of (12) and the assumption $\lim _{x \rightarrow \infty} \psi_{1}(x)[1-F(x)]^{n}=0$, we have

$$
\int_{t}^{\infty} \psi_{1}^{\prime}(x)(1-F(x))^{n} d x=-\left[\psi_{1}(t)-q_{1}(t)\right](1-F(t))^{n}
$$

Differentiating both sides of the above equation with respect to $t$, we arrive at

$$
\frac{f(t)}{1-F(t)}=\frac{-q_{1}^{\prime}(t)}{n\left[\psi_{1}(t)-q_{1}(t)\right]}, \quad t>0
$$

Now, integrating (14) from 0 to $x$, we have, in view of $\int_{0}^{\infty} \frac{-q_{1}^{\prime}(t)}{\left[\psi_{1}(t)-q_{1}(t)\right]} d t=\infty$, a $c d f F$ given by (13).

Remarks 2.3.2. (c) Taking, e.g.,

$$
\psi_{1}(x)=\left\{1-p\left(1-e^{-\beta x}\right)^{\alpha}\right\}^{n+1} \text { and } q_{1}(x)=(1-p)\left\{1-p\left(1-e^{-\beta x}\right)^{\alpha}\right\}^{n}
$$

in Proposition 2.3.1, equation (14) will be

$$
\frac{f(x)}{1-F(x)}=\frac{(1-p) \alpha \beta e^{-\beta x}\left(1-e^{-\beta x}\right)^{\alpha-1}}{\left\{1-p\left(1-e^{-\beta x}\right)^{\alpha}\right\}\left\{1-\left(1-e^{-\beta x}\right)^{\alpha}\right\}} .
$$

Rewriting (15), we have

$$
\frac{f(x)}{1-F(x)}=\left[\frac{\alpha \beta e^{-\beta x}\left(1-e^{-\beta x}\right)^{\alpha-1}}{1-\left(1-e^{-\beta x}\right)^{\alpha}}-\frac{p \alpha \beta e^{-\beta x}\left(1-e^{-\beta x}\right)^{\alpha-1}}{1-p\left(1-e^{-\beta x}\right)^{\alpha}}\right],
$$

from which, in view of (13), we have $F(x)=1-\frac{1-\left(1-e^{-\beta x}\right)^{\alpha}}{1-p\left(1-e^{-\beta x}\right)^{\alpha}}$, which is $c d f$ of NGEG. 
(d) Taking, e.g., $\psi_{1}(x)=2 \gamma\left\{-\ln \left[1-e^{-\left(\frac{x}{\beta}\right)^{k}}\right]^{\alpha}, \delta\right\}$ and $q_{1}(x)=\frac{1}{2} \psi_{1}(x)$ in Proposition 2.3.1, equation (14) will be

$$
\frac{f(x)}{1-F(x)}=\frac{-\frac{d}{d x} \gamma\left\{-\ln \left[1-e^{-\left(\frac{x}{\beta}\right)^{k}}\right]^{\alpha}, \delta\right\}}{\gamma\left\{-\ln \left[1-e^{-\left(\frac{x}{\beta}\right)^{k}}\right]^{\alpha}, \delta\right\}},
$$

from which,

$$
F(x)=1-\frac{\gamma\left\{-\ln \left[1-e^{-\left(\frac{x}{\beta}\right)^{k}}\right]^{\alpha}, \delta\right\}}{\Gamma(\delta)}, \quad x \geq 0
$$

which is $c d f$ of GEW.

\section{References}

[1] Bidram, H., Behboodian, J. and Towhidi, M., The beta Weibull-geometric distribution, J. Statistical Computation and Simulation, iFirst, (2011), 1-16.

[2] Bidram, H., Behboodian, J. and Towhidi, M., A new generalized exponential geometric distribution. To appear in Commun. in Statist. Theory-Method.

[3] Galambos, J. and Kotz, S., Characterizations of probability distributions. A unified approach with an emphasis on exponential and related models, Lecture Notes in Mathematics, 675, Springer, Berlin, 1978.

[4] Glänzel, W., A characterization of the normal distribution, Studia Sci. Math. Hungar. 23 (1988), 89-91.

[5] Glänzel, W., A characterization theorem based on truncated moments and its application to some distribution families, Mathematical Statistics and Probability Theory (Bad Tatzmannsdorf, 1986), Vol. B, Reidel, Dordrecht, 1987, 75-84.

[6] Glänzel, W., Some consequences of a characterization theorem based on truncated moments, Statistics 21 (1990), 613-618.

[7] Glänzel, W., IrWin, J., A characterization tool for discrete distributions under Window(R), Proc. COMPSTAT' 94 (Vienna, 1994), Short Communications in Computational Statistics, ed. by R. Dutter and W. Grossman, Vienna, 199-200.

[8] Glänzel, W., Telcs, A. and Schubert, A., Characterization by truncated moments and its application to Pearson-type distributions, Z. Wahrsch. Verw. Gebiete 66 (1984), 173-183.

[9] Glänzel, W. and Hamedani, G.G., Characterizations of univariate continuous distributions, Studia Sci. Math. Hungar. 37 (2001), 83-118.

[10] Hamedani, G.G., Characterizations of Cauchy, normal and uniform distributions, Studia Sci. Math. Hungar. 28 (1993), 243-247.

[11] Hamedani, G.G., Characterizations of univariate continuous distributions. II, Studia Sci. Math. Hungar. 39 (2002), 407-424.

[12] Hamedani, G.G., Characterizations of univariate continuous distributions. III, Studia Sci. Math. Hungar. 43 (2006), 361-385.

[13] Hamedani, G.G. and Ahsanullah, M., Characterizations of Weibull geometric distribution, J. of Statistical Theory \& Applications, 10 (2011), 581-590.

[14] Kotz, S. and Shanbhag, D.N., Some new approaches to probability distributions, Adv. in Appl. Probab. 12 (1980), 903-921. 


\section{G.G. Hamedani}

[15] Ortega, E.M.M., Cordeiro, G.M. and Pascoa, A.R., The generalized gamma geometric distribution, to appear in J. of Statistical Theory \& Applications, 10 (2011), 433-454.

[16] Pinho, L.G.B., Cordeiro, G.M. and Nobre, J.S., The Gamma-exponentiated Weibull distribution. Submitted for publication. 